\title{
COMPUTATIONAL MODELING APPLIED TO THE STUDY OF THERMAL BUCKLING OF COLUMNS
}

\author{
R. da S. Michaello ${ }^{\mathrm{a}}$, \\ D. Helbig', \\ L. A. O. Rochab, \\ M. de V. Real ${ }^{c}$, \\ E. D. dos Santos ${ }^{c}$, \\ and L. A. Isoldi \\ ${ }^{a}$ Universidade Federal do Rio Grande \\ Programa de Pós-Graduação em \\ Modelagem Computacional \\ Avenida Itália, km 8, CP. 474, \\ Rio Grande, RS, Brasil \\ robertaa@rocketmail.com \\ ${ }^{\mathrm{b}}$ Universidade Federal do Rio Grande do Sul \\ Programa de Pós-Graduação em \\ Engenharia Mecânica \\ Rua Sarmento Leite, 425, $2^{\circ}$ Andar, \\ CEP 90.050-170, Porto Alegre, RS, Brasil \\ ${ }^{\mathrm{c}}$ Universidade Federal do Rio Grande - \\ Programa de Pós-Graduação em \\ Engenharia Oceânica \\ Avenida Itália, km 8, CP. 474, \\ Rio Grande, RS, Brasil \\ Received: March 04, 2015 \\ Revised: April 06, 2015 \\ Accepted: May 18, 2015
}

ABSTRACT

Buckling is an instability phenomenon that can happen in slender structural components when subjected to a compressive axial load. This phenomenon can occur due to an externally applied force, which when exceed a certain limit, called critical load, will promote the mechanical buckling on the structural member. Another buckling possibility happens to statically indeterminate structural elements when submitted to a positive temperature variation. As the axial displacements are restricted, if the temperature gradient is larger than the critical temperature variation, it will be generated a compressive axial load higher than the critical load of the structural component and the thermal buckling will occur. In this context, the present work presents a computational model to solve the thermal buckling problem of columns. A thin shell finite element, called SHELL93, was adopted for the computational domain discretization. It was employed a solution involving homogeneous algebraic equations, where the critical temperature variation is determined by the smallest eigenvalue and the buckled configuration is defined by its associated eigenvector. A case study was performed considering a steel column with three different support conditions at its ends: fixed-fixed, fixed-pinned, and pinned-pinned. The numerical results obtained for the critical temperature variation showed a maximum absolute difference around $2 \%$ when compared to the analytical solutions. Moreover, the buckled shape of the column, for each case, was defined in agreement with the configurations found in literature. Therefore, the computational model was verified, i.e., it is able to satisfactorily predict the mechanical behavior of the thermal buckling of columns. So, it is possible to use this numerical model in practical situations that do not have an analytical solution, as is the case of the thermal buckling of columns with cutouts.

Keywords: thermal buckling, columns, finite element method (FEM)

\section{NOMENCLATURE}

A cross sectional area of the bar, $\mathrm{mm}^{2}$

$b$ flange width, mm

d profile height, $\mathrm{mm}$

e web thickness, mm

E Young' modulus, GPa

I moments of inertia, $\mathrm{mm}^{4}$

$\mathrm{L} \quad$ length of the slender bar, $\mathrm{m}$

$\mathrm{P}$ support reactions, $\mathrm{N}$

$r \quad$ rotation about the axis

$t \quad$ flange thickness, mm

$\Delta \mathrm{T}$ temperature variation, ${ }^{\circ} \mathrm{C}$

$u \quad$ translation

\section{Greek symbols}

$\alpha \quad$ coefficient of thermal expansion, ${ }^{\circ} \mathrm{C}^{-1}$

$\delta_{\mathrm{A} / \mathrm{B}}$ displacement of end $\mathrm{A}$ relative to the end $\mathrm{B}$ of the bar, $m$
$\delta_{\mathrm{P}} \quad$ length variation caused by the support reaction $\mathrm{P}, \mathrm{m}$

$\delta_{\mathrm{T}} \quad$ length variation caused by temperature gradient $\Delta \mathrm{T}, \mathrm{m}$

$\varepsilon_{\mathrm{T}}$ thermal normal strain of the bar

$v \quad$ Poisson's ratio

$\pi \quad$ mathematical constant

$\begin{array}{ll}\text { Subscripts } \\ \text { cr } & \text { critical } \\ \text { X } & \text { X axis } \\ \text { y } & \text { y axis } \\ \text { Z } & \text { Z axis }\end{array}$

\section{INTRODUCTION}

Slender structural elements, as the steel columns, are widely used for the construction of naval and offshore structures. Examples of these 
applications can be observed in Figs. 1a (http://pbrasil.wordpress.com/2010/06/02/de-olho-nanoticia-setores-aeronautico-e-naval-precisam-de-inve stimentos-continuos-dizem-especialistas-na-ci/, access: 15/05/2015) and 1b (http://jornalcanal16. com /?p=11159, access: 15/05/2015) where, respectively, a ship hull structure and a self-elevating platform for petroleum exploration are shown.
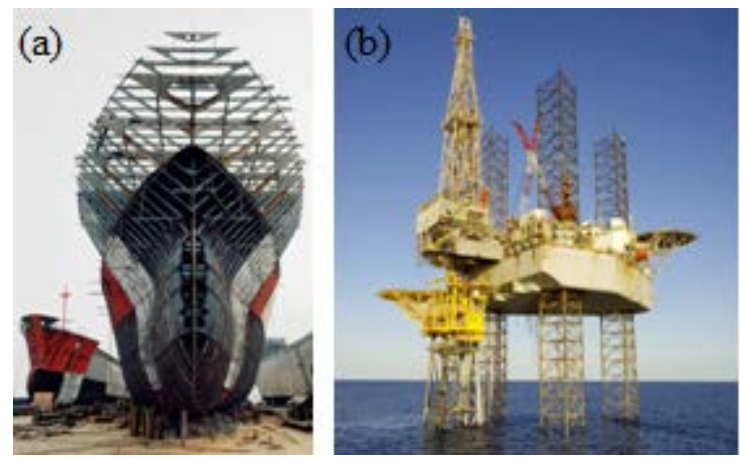

Figure 1. Steel column applications: (a) ship hull structure; and (b) self-elevating platform.

In accordance with Iyengar (1988), a structural component can present two failure types: of material and of shape. In the material failure, the component stress exceeds the specified safe limit, resulting in its collapse; while in the shape failure, even if the stress is below to the safe limit, the component is not capable of maintain its original shape. The shape failure depends of the component geometry as well as of the applied load, being possible to occur in slender structural elements subjected to compressive axial loads.

Therefore, this shape failure is defined as a structural instability phenomenon and is known as buckling. While the axial compressive load applied in a slender structural component is relatively small, the increase of its magnitude will only result in a normal strain and hence in the decreasing of the component length. However, when a certain load level is reached, the structural component will suffer an abrupt lateral bending. This load level is called critical load and this lateral bending originates large deformations, causing the collapse of the structural element (Chajes, 1974).

Normally, a compressive stress in a structural member is caused by a pure axial compressive load, by a bending moment, by a shear or local concentrated loads, or by a combination among these (Åkesson, 2007). However, there is still a possibility of a structural component to suffer a compressive stress due to the temperature elevation, since the axial displacements of the component ends are constrained by its support conditions (Hibbeler, 2010).

Therefore, if the axial displacements of a structural element are restrained and this component is subjected to a positive variation (increase) of temperature, will surge an internal compressive force which, depending of its magnitude, can cause buckling, being, in this case, called thermal buckling.

In this context, the goal of this work is to develop a computational model to solve the thermal buckling problem of steel columns, aiming to determine the critical temperature variation, i.e., the temperature variation that causes this phenomenon. To do so, the software ANSYS, which is based on the Finite Element Method (FEM), was employed. Different support conditions for the column were considered. The numerical model was verified, comparing its results with the thermal buckling solutions obtained analytically.

\section{THERMAL BUCKING}

The thermal expansion of a structural component, depending of the support conditions of its ends, can cause a compressive stress, with the possibility of occurrence of an elastic instability: the thermal buckling.

In agreement with Beer and Jonston (1995) and Cassenti (2012), to understand how the thermal buckling occurs in a column, it is necessary to take into account a slender bar with length $L$, made of homogeneous material and with constant cross sectional area, which is, initially, supported by a horizontal flat surface, as it can be viewed in Fig. 2a.

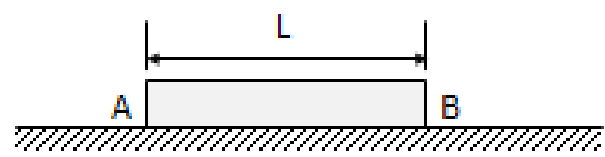

(a)

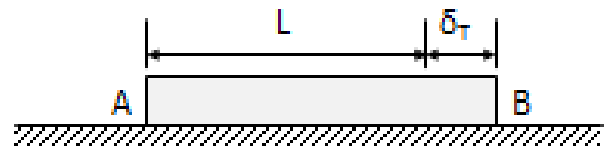

(b)

Figure 2. Bar supported by a flat horizontal surface:

(a) without temperature variation; and (b) with temperature variation.

If the bar is exposed to a positive temperature variation $\Delta T$, as in Fig. $2 \mathrm{~b}$, it will suffer an elongation $\delta_{T}$ proportional both to the temperature variation and to the bar length:

$$
\delta_{T}=\alpha \Delta T L
$$

where: $\alpha$ is the coefficient of thermal expansion. If the length variation of the bar, defined by Eq. (1), is related with the initial length of the bar, it is possible to determine the thermal normal strain of the bar $\varepsilon_{T}$ :

$$
\varepsilon_{T}=\frac{\delta_{T}}{L}=\alpha \Delta T
$$


In this case, the bar do not have any type of support restraining its normal strain, hence there is no thermal stress associated. However, if the same bar of Fig. 2 has its ends fixed, as showed in Fig. 3a, and is submitted to a positive temperature variation $\Delta T$, support reactions $P$ contrary to the expansion movement (normal displacement) of the bar will arise, due the presence of the supports, as illustrated in Fig. 3b.
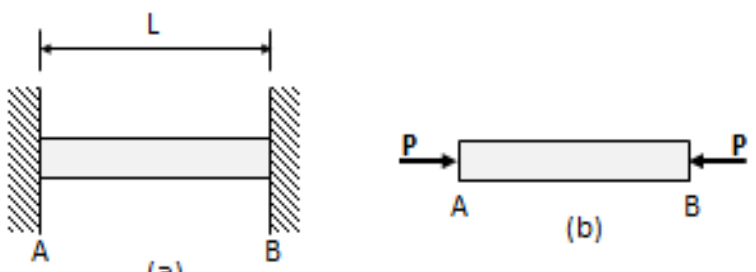

(a)

(b)

Figure 3. Bar fixed-fixed: (a) submitted to the positive temperature variation; and (b) support reactions generated.

As the bar is fixed-fixed, it results in a statically indeterminate structure, i.e., it is not possible to define its support reactions only using the equilibrium equations. So, it is necessary to consider a displacement compatibility equation (Hibbeler, 2010):

$$
\delta_{A / B}=\delta_{T}-\delta_{P}=0
$$

where: $\delta_{A / B}$ is the displacement of end $\mathrm{A}$ relative to the end $\mathrm{B}$ of the bar and $\delta_{P}$ is the length variation caused by the support reaction $P$, given by:

$$
\delta_{P}=\frac{P L}{A E}
$$

where: $A$ is the cross sectional area of the bar and $E$ is the Young's modulus.

Thus, replacing Eqs. (2) and (4) in Eq. (3), it is possible to determine the magnitude of the support reaction $P$ generated by the temperature variation:

$$
P=A E \alpha \Delta T
$$

Whereas that for a fixed-fixed column its buckling critical load is defined by (Hibbeler, 2010):

$$
P_{c r}=\frac{4 \pi^{2} E I}{L^{2}}
$$

and equating the load imposed to the bar due to the thermal variation, given by Eq. (5), to the buckling critical load, obtained from Eq. (6), it is possible to define the critical temperature variation, i.e., the temperature variation which causes the thermal buckling (Beer and Johnston, 1995; Cassenti, 2012):

$$
\Delta T_{c r}=\frac{4 \pi^{2} I}{\alpha A L^{2}}
$$

Following analogous procedure, one can obtain analytical solutions for the critical temperature variation for a fixed-pinned column:

$$
\Delta T_{c r}=\frac{2.0457 \pi^{2} I}{\alpha A L^{2}}
$$

and for a pinned-pinned column:

$$
\Delta T_{c r}=\frac{\pi^{2} I}{\alpha A L^{2}}
$$

\section{COMPUTATIONAL MODELING}

For the numerical determination of the critical temperature variation, i.e., the temperature variation responsible for the thermal buckling of slender columns it was developed a computational model in the ANSYS software. ANSYS is a computational tool which employ as discretization method the Finite Element Method (FEM), allowing linear and nonlinear analyses in static and dynamic structural problems (ANSYS, 2005).

In general terms, the FEM is based on the division of the integration domain, continuous, in a finite number of small regions called finite elements, turning the continuous medium into discrete. The behavior of each element is arbitrated in a approximate way, with the proviso that the mesh generated by the finite elements behaves similarly to the original continuous. At the so-called displacement model of the FEM, the nodal displacement field are arbitrated and, hence, the interaction of the stress components among adjacent elements is replaced by the interaction of the nodal forces among elements. In this way, the infinitesimal equilibrium considered in the mathematical model of the continuous medium is replaced by the equilibrium of each singly finite element, transforming the differential equilibrium equations into algebraic equilibrium equations of the element as a whole. From these algebraic equations defined for each finite element, a system of equilibrium equations of the elements mesh is obtained. This global system, after the inclusion of the boundary conditions of the problem, allows the solution determination in terms of the nodal displacements (Madenci and Guven, 2006).

For the computational model used in the present work it was adopted the SHELL93 finite element, because it is indicated for the numerical simulation of thin plates and shells. The SHELL93 element, shown in Fig. 4, has eight nodes and six degrees of freedom per node, i.e., three translations in $x, y$, and $z$ directions $\left(u_{x}, u_{y}\right.$ e $\left.u_{z}\right)$ and three rotations about the $x$, 
$y$, and $z$ axis $\left(r_{x}, r_{y}\right.$ e $\left.r_{z}\right)$, totaling twenty four degrees of freedom per element (ANSYS, 2005).

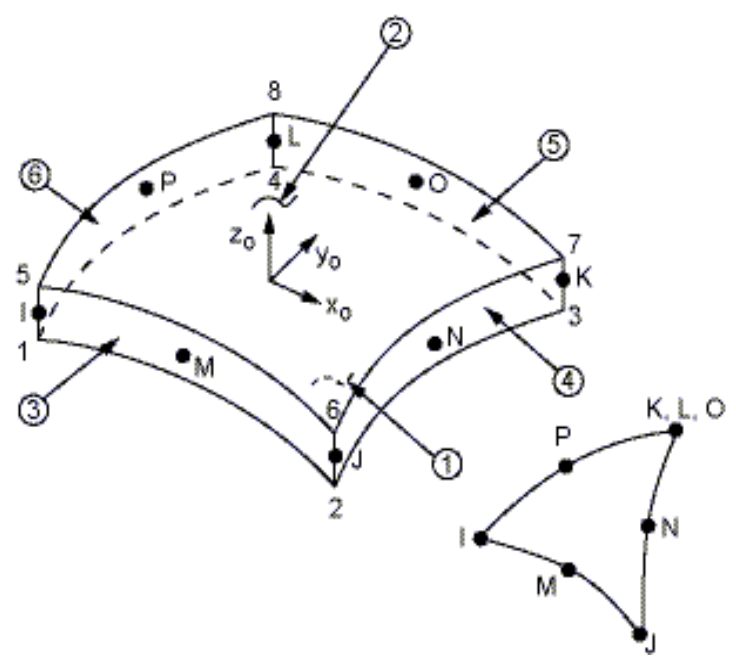

Figure 4. Finite element SHELL93.

For the numeric study of the thermal buckling phenomenon it was adopted the eigenvalueeigenvector analysis. The finite element equilibrium equations for this analysis type involves the solution of homogeneous algebraic equations whose smallest eigenvalue corresponds to the critical buckling temperature and the associated eigenvector represents the first buckling mode of the structure. This solution is analogous to one used to determine the mechanical critical buckling load of a column, where the smallest eigenvalue indicates de critical load magnitude and the associated eigenvector defines the geometric configuration of the first buckling mode (Madenci and Guven, 2006). Therefore, the difference between the reference temperature and the temperature applied on the column should be only $1^{\circ} \mathrm{C}$, similarly to what is done in the mechanical analysis where the eigenvalues and eigenvectors are calculated for a unitary applied load. So, with this difference of $1^{\circ} \mathrm{C}$, the first eigenvalue will determine the critical temperature variation which causes the thermal buckling (Cassenti, 2012). In the ANSYS software the eigenvalue-eigenvector problem is solved by means the Lanczos numerical method (ANSYS, 2005).

\section{RESULTS AND DISCUSSION}

To show the efficacy of the proposed computational model, a case study was developed. The A-36 steel W310×21 member shown in Fig. 5, with Young' modulus $E=210 \mathrm{GPa}$, Poisson's ratio $v$ $=0.3$, and coefficient of thermal expansion $\alpha=$ $12 \times 10^{-6}{ }^{\circ} \mathrm{C}^{-1}$, is considered here as a fixed-fixed column type structure, having the following geometric characteristics: length $L=4.5 \mathrm{~m}$, height $d$ $=303 \mathrm{~mm}$, flange thickness $t=5.7 \mathrm{~mm}$, flange width $b=101 \mathrm{~mm}$, web thickness $e=5.08 \mathrm{~mm}$, cross sectional area $A=2680 \mathrm{~mm}^{2}$, and moments of inertia $I_{z}=37 \times 10^{6} \mathrm{~mm}^{4}$ and $I_{y}=0.986 \times 10^{6} \mathrm{~mm}^{4}$.

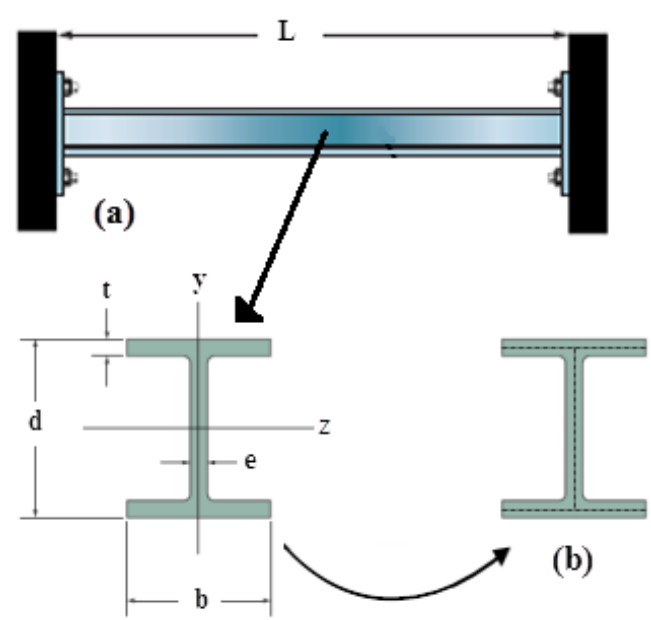

Figure 5. Case study: (a) fixed-fixed steel column; and (b) simplification hypothesis.

As the moment of inertia about $y$ axis is lower than the moment of inertia about $z$, the thermal buckling will occur about the $y$ axis. Therefore, from Eq. (7) it is possible to determine the analytical value for the critical temperature of $\Delta T_{c r}=59.77^{\circ} \mathrm{C}$.

Other column support conditions were also studied: fixed-pinned (Fig. 6a) and pinned-pinned (Fig. 6b), resulting $\Delta T_{c r}=30.57{ }^{\circ} \mathrm{C}$ and $\Delta T_{c r}=14.94$ ${ }^{\circ} \mathrm{C}$, respectively, from the analytical solutions according to Eqs. (8) and (9). It is important to highlight that the pinned ends of the columns have this support condition both in $y$ axis as in $z$ axis, although this feature cannot be observed in Fig. 6 .

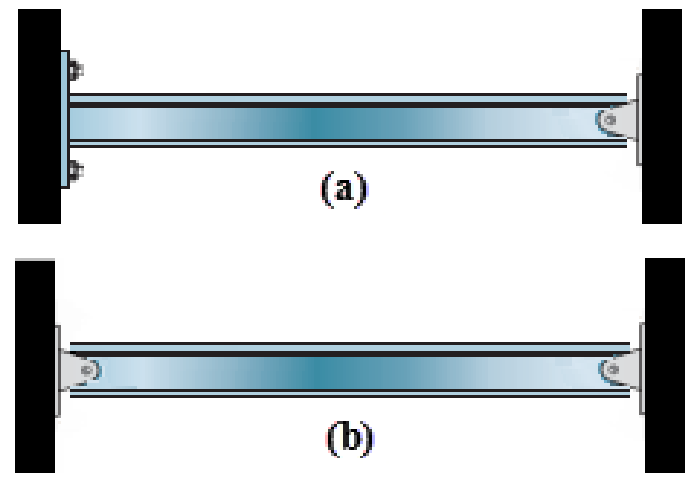

Figure 6. Structural element binding: (a) fixed-pinned and (b) pinned-pinned.

In addition, for the numerical solution of the problem, as the finite element employed on the computational model is a shell type element, a simplification was taken into account for the computational domain construction: the flanges and the web of the steel column were represented by its middle surfaces, as indicated by the dashed lines in Fig. 5b. In all performed numerical simulations, the 
computational domain discretization was made with a regular mesh generated with quadrilaterals elements having characteristic length of $10 \mathrm{~mm}$, as can be viewed in Fig. 7. The adopted reference temperature was $25^{\circ} \mathrm{C}$ and the applied temperature, as already explained, was then $26^{\circ} \mathrm{C}$.

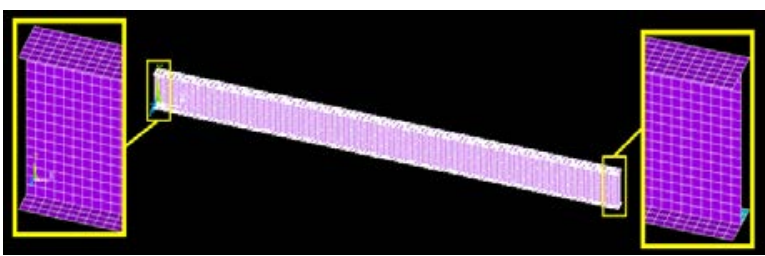

Figure 7. Computational domain discretization.

For the case of the fixed-fixed column, the boundary conditions considered at its ends were: $u_{x}=$ $u_{y}=u_{z}=r_{x}=r_{y}=r_{z}=0$, applied at the lines which represent the flanges and web of the column (dashed lines in Fig. 5b). It was numerically obtained a critical temperature variation of $\Delta T_{c r}=58.46{ }^{\circ} \mathrm{C}$, presenting a relative difference to the analytical result of $-2.19 \%$. The buckled configuration of the column, in this case, have its maximum deflection at the central region (red region in Fig. 8) in the $z$ direction, while its ends remain without any displacements or rotations.

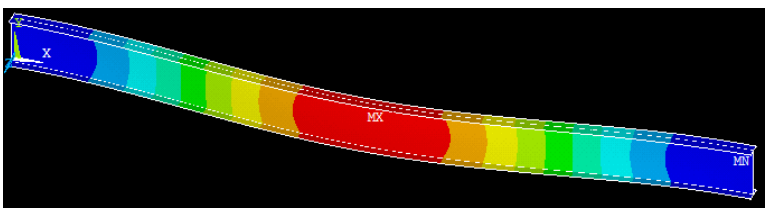

Figure 8. Thermal buckling of the fixed-fixed column.

For the fixed-pinned column, it were imposed as boundary conditions: $u_{x}=u_{y}=u_{z}=r_{x}=r_{y}=r_{z}=0$, applied on the lines at the left (fixed) end of the computational domain; and $u_{y}=r_{x}=r_{z}=0$ on the flanges lines and $u_{x}=u_{y}=u_{z}=r_{x}=r_{z}=0$ on the web line, applied at the right (pinned) end. The critical temperature variation defined by means the numerical simulation was $\Delta T_{c r}=30.61{ }^{\circ} \mathrm{C}$, presenting a relative difference of only $0.13 \%$ when compared with the corresponding analytical solution. The column shape after the thermal buckling is depicted in Fig. 9.

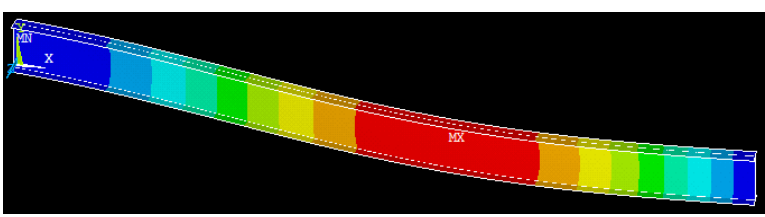

Figure 9. Thermal buckling of the fixed-pinned column.
Due to the greater mobility allowed to the right end of the column by the pinned support condition, when compared with the fixed-fixed case, one can note in Fig. 9 that the maximum deflection suffered by the component (in red color) do not occurs in its central region as earlier observed in Fig. 8, but in a region located between its central region and its pinned end. It is worth to mention that this deformed configuration is in agreement with the buckled shape of a fixed-pinned column (Hibbeler, 2010).

Finally, the numerical simulation for the thermal buckling of a pinned-pinned column was carried out, being imposed at both ends the follow boundary conditions: $u_{y}=r_{x}=r_{z}=0$ on the flanges lines and $u_{x}=u_{y}=u_{z}=r_{x}=r_{z}=0$ on the web lines. A value of $\Delta T_{c r}=15.18{ }^{\circ} \mathrm{C}$ was defined by the computational modeling, having a difference of $1.61 \%$ when compared with the analytical solution. The deformed configuration of the pinned-pinned column due the thermal buckling phenomenon is presented in Fig. 10. Here, as in Fig. 8, owing to the symmetry of the support conditions at the column ends, the maximum deflection occurs on its central region (red color in Fig 10).

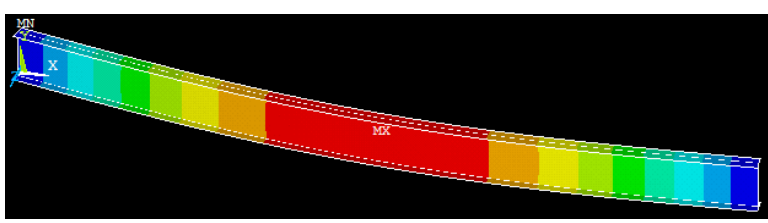

Figure 10. Thermal buckling of the pinned-pinned column.

Analyzing the numerical results quantitatively, it was found a maximum absolute difference around $2 \%$ in comparison with the analytical solutions, verifying the proposed computational model. Besides, if a qualitative analysis is performed, one can observe that the computational model is able to predict the thermally buckled shape of a column in agreement with the literature indications (Hibbeler, 2010; Barron and Barron, 2012), proving the efficacy of the proposed model.

\section{CONCLUSIONS}

In this work it was presented a computational model capable of solving the thermal buckling problem of slender columns with different support conditions. The ANSYS software, which is based on the Finite Element Method (FEM), was employed, being used the SHELL93 element for the computational domain discretization. The eigenvalueeigenvector analysis was adopted, determining with the first eigenvalue the critical temperature variation, i.e., the temperature variation that causes the thermal buckling; and with the first eigenvector the first buckling mode, i.e., the buckled configuration of the column. To do so, it was imposed a difference of $1^{\circ} \mathrm{C}$ 
between the reference temperature and the applied temperature over the column.

The numerical results are in agreement with the analytical solutions. It has been found a maximum absolute difference of approximately $2 \%$ between numerical and analytical results. Moreover, the deflections suffered by the columns due the thermal buckling, for each studied support condition, are also in agreement with those indicated in literature. Therefore, one can consider that the proposed computational model was verified and that it is capable to solve the thermal buckling problem of a column in an adequate way.

In addition, as already expected, it was observed that when the column has support conditions more restrictive, it is necessary more elevated temperature variations to promote the thermal buckling. It is need a $\Delta T_{c r}$ of almost $60{ }^{\circ} \mathrm{C}$ to cause the thermal buckling on the fixed-fixed column, while for the pinnedpinned column a $\Delta T_{c r}$ only around $15{ }^{\circ} \mathrm{C}$ causes the thermal buckling phenomenon.

It is worth to mention thatk from this computational model, it will be possible to study practical situations which do not have analytical solutions, such as to analyze the effect of distributed cutouts along the column over the thermal buckling, justifying the continuity of this research.

\section{ACKNOWLEDGEMENTS}

The authors would like to thank to the Universidade Federal do Rio Grande (FURG), Universidade Federal do Rio Grande do Sul (UFRGS), and Conselho Nacional de Desenvolvimento Científico e Tecnológico (CNPq) for their support.

\section{REFERENCES}

Åkesson, B., 2007, Plate Buckling in Bridges and Other Structure, Taylor \& Francis.

Ansys, 2005, User's Manual (version 10.0), Swanson Analysis System Inc.

Barron, R. F., and Barron, B. R., 2012, Design for Thermal Stresses, John Wiley \& Sons.

Beer, F. P., and Johnston, E. R. Jr., 1995, Resistência dos Materiais, Makron Books. (in Portuguese)

Cassenti, B., 2012, Thermal Buckling of a 2D Beam Fixed at Both Ends, University of Connecticut.

Chajes, A., 1974, Principles of Structural Stability Theory, Prentice Hall.

Hibbeler, R. C., 2010, Resistência dos Materiais, Pearson Prentice Hall. (in Portuguese)

Iyengar, N. G. R., 1988, Structural Stability of Columns and Plates, John Wiley \& Sons.

Madenci, E., and Guven, I., 2006, The Finite Element Method and Applications in Engineering using ANSYS, Springer. 